A N N A L E S Annales de Bretagne et des Pays de l'Ouest

Anjou. Maine. Poitou-Charente. Touraine

109-4 | 2002

Les étrangers dans l'Ouest de la France (XVIII $-\mathrm{XXX}^{\mathrm{e}}$ siècle)

\title{
Soldats américains et civils français dans la région de la Basse-Loire pendant la Première Guerre mondiale
}

\section{Yves-Henri Nouailhatn}

\section{(2) OpenEdition \\ Journals}

Édition électronique

URL : http://journals.openedition.org/abpo/1527

DOI : 10.4000/abpo. 1527

ISBN : 978-2-7535-1488-1

ISSN : 2108-6443

Éditeur

Presses universitaires de Rennes

Édition imprimée

Date de publication : 20 décembre 2002

Pagination : 177-190

ISBN : 978-2-86847-794-1

ISSN : 0399-0826

Référence électronique

Yves-Henri Nouailhatn, «Soldats américains et civils français dans la région de la Basse-Loire pendant la Première Guerre mondiale », Annales de Bretagne et des Pays de l'Ouest [En ligne], 109-4 | 2002, mis en ligne le 20 décembre 2004, consulté le 01 mai 2019. URL : http://journals.openedition.org/ abpo/1527; DOI : 10.4000/abpo.1527 


\title{
Soldats américains et civils français dans la région de la Basse-Loire pendant la Première Guerre mondiale
}

\author{
Yves-Henri NOUAILHAT \\ Professeur émérite d'histoire contemporaine à l'Université de Nantes \\ Centre de Recherche sur l'Histoire du Monde Atlantique
}

Le 6 avril 1917, le Congrès des États-Unis, sur proposition du président Wilson, déclare la guerre à l'Allemagne. Les raisons de cette entrée en guerre sont bien connues : la décision allemande de déclencher la guerre sous-marine à outrance s'en prend directement aux intérêts américains. Lorsque les sous-marins allemands commencent à couler des navires américains, Wilson ne réagit pas seulement pour défendre les droits des neutres et la liberté des mers. Il sait que seule une réaction vigoureuse peut sauver le prestige des États-Unis et leur donner des chances d'influencer la paix. L'entrée en guerre des États-Unis allait en effet permettre au président américain, qui est persuadé qu'il faut réformer profondément le système des relations internationales, de mettre fin plus rapidement au conflit et de mettre en place un nouvel ordre international propre à éviter les guerres dans l'avenir.

Cependant, au lendemain de leur entrée en guerre, les Américains sont dans l'incapacité d'envoyer immédiatement un corps expéditionnaire en France pour la simple raison que leur armée est presque inexistante. Or, au cours de sa mission aux États-Unis en avril-mai 1917, le maréchal Joffre évoque la nécessité de mettre sur pied " une grande armée américaine " et, en attendant que cette armée soit prête, émet le souhait que certains éléments puissent être envoyés rapidement afin d'apporter un appui moral en attendant l'appui matériel et massif espéré. Un corps expéditionnaire d'une vingtaine de milliers de combattants est donc placé sous le commandement du général Pershing et la région de la Basse-Loire devient le siège de la base $\mathrm{n}^{\circ} 1$ des American Expeditionary Forces, en raison des capacités d'accueil des ports de Saint-Nazaire et Nantes. Les ports de la Manche étaient encombrés, les ports de la Méditerranée trop éloignés. D'autres ports seront également utilisés en particulier Bordeaux, siège de la base $\mathrm{n}^{\circ} 2$, Brest (base $\mathrm{n}^{\circ}$ ) et La Rochelle-La Pallice (base $\mathrm{n}^{\circ}$ ). 
Les premières troupes américaines débarquent à Saint-Nazaire le 26 juin 1917. Au départ, la base $n^{\circ} 1$ comprenait huit départements. À partir de juin 1918, elle n'en compte plus que cinq : Loire-Inférieure, Morbihan, Maineet-Loire, Vendée et Deux-Sèvres, mais, en fait, son activité est presque uniquement concentrée dans la Basse-Loire. Cette base occupe une place importante dans le dispositif américain de l'arrière. En effet, la voie ferrée Saint-Nazaire, Nantes, Tours, Bourges, Nevers, Dijon, Is-sur-Tille permettra d'acheminer le matériel jusqu'à la zone prévue pour le corps expéditionnaire sur le front. Les ports de la Basse-Loire sont utilisés pour le débarquement de matériel - au total entre avril 1917 et octobre 1918, 3300000 tonnes ont été débarquées, soit à peu près le tiers du matériel américain envoyé en France pendant cette période - et des hommes - au total 198000 hommes débarquent à Saint-Nazaire en 1917 et 1918. La cité nazairienne sert aussi de port de rembarquement pour les soldats américains en 1919 : 478000 soldats sont repartis par Saint-Nazaire. Si l'on considère l'ensemble des localités de Loire-Inférieure où sont installés des Américains, on peut dire que le chiffre total se situe entre 15000 et 60000 selon les moments, la quasi totalité se trouvant à Saint-Nazaire, Montoir, Savenay et Nantes. Une partie des soldats est constituée de troupes de passage - celles qui viennent de débarquer ou celles qui vont rembarquer - les autres sont formées de troupes "sédentaires " dont le rôle est d'assurer le fonctionnement des diverses installations. Étant donné la modestie des chiffres de population de certaines localités, le nombre des Américains présents égale ou dépasse le chiffre de la population, notamment à Savenay, Montoir ou Saint-Nazaire ${ }^{1}$.

La présence américaine est bien visible. Dans l'ensemble, entre juin 1917 et novembre 1918, les relations entre la population française et les soldats américains sont bonnes : on peut même parler d'un véritable engouement des Français pour les Sammies. Elles se dégradent à partir de l'armistice. Si l'on dresse un bilan général, on constate finalement que l'influence de la présence américaine a été bien limitée.

\section{Une présence bien " visible "}

Les ports de Saint-Nazaire et de Nantes accueillent de nombreux navires d'outre Atlantique. Il n'est pas rare de voir arriver à Saint-Nazaire dix ou même vingt navires américains à la fois. Une grande animation règne dans la ville : les Doughboys, que les Français ont surnommé Sammies flânent dans les rues, en général par petits groupes, mangeant cerises ou groseilles ou dévalisant les pâtisseries. Les allées et venues des véhicules militaires - camions ou automobiles - impressionnent la population. Il ne faut pas oublier que Nantes et surtout Saint-Nazaire possédaient en 1917 un parc automobile fort réduit, sans doute quelques dizaines. Nazairiens et Nantais n'avaient vu aucun camion militaire avant juin 1917.

1. NouAlLHAT, Yves-Henri, Les Américains à Nantes et à Saint-Nazaire, Paris, Les Belles Lettres, 1972. 
Les Américains sont partout. Ils ont occupé pour ainsi dire tous les établissements disponibles et tous les bâtiments utilisables pour installer différents services, des hôpitaux ou stocker du matériel. Ces installations sont éparpillées, variées et de tailles diverses. La réalisation la plus importante fut sans conteste le camp de Montoir avec ses 138 baraquements, ses 200 kilomètres de voie ferrée et ses gares de triage. On peut également citer la construction de deux petits barrages : celui du Bois Joalland, permettant la réalisation d'un bassin de retenue de 2 millions de $\mathrm{m}^{3}$ pour l'alimentation en eau de Saint-Nazaire, qui a servi jusqu'en 1960; celui de la vallée Mabile de $400000 \mathrm{~m}^{3}$ près de Savenay pour fournir de l'eau au grand hôpital aménagé dans l'École normale d'instituteurs de Savenay ${ }^{2}$. La voie ferrée est doublée dans la traversée de Nantes. À Saint-Nazaire, d'importants travaux ferroviaires permettent d'améliorer la desserte des quais, dotés en outre de nouvelles grues. Une activité particulièrement remarquable des Américains à Saint-Nazaire fut le montage de locomotives qu'ils réalisèrent dans les locaux des Forges et Chantiers de la Loire. Quant à l'atelier de réparation des wagons, il traita du $1^{\mathrm{er}}$ août au 11 novembre 1918, 3000 wagons français et 1400 américains. De leur côté, les ateliers de réparation automobile du camp $\mathrm{n}^{\circ} 8$ révisèrent ou réparèrent 20000 véhicules. À Nantes, deux importants ateliers de réparation automobile sont installés au Champ de Mars et au Parc des Sports. Un terrain d'aviation est aménagé au champ de manœuvre militaire du Bêle.

En dehors des aspects matériels de la présence américaine, on peut évoquer les nombreuses occasions de rencontre entre soldats américains et civils français : cérémonies en l'honneur de l'Independance Day, les 4 juillet 1917 et 1918, commémorations de la naissance d'Abraham Lincoln, les 12 février 1917 et 1918, marquées par des discours, des banquets, des conférences, des défilés ou des concerts; célébration du Thanksgiving ou de Noël. Ainsi le 25 décembre 1917, les Américains invitent dans leur camp 1200 à 1300 enfants de 7 à 13 ans parmi les 6000 à 7000 élèves des écoles primaires de Saint-Nazaire à un arbre de Noël pour leur distribuer des cadeaux. Deux mille parents assistent à cette journée. " Nous souhaitons divertir les enfants pour leur faire plaisir et démontrer notre amitié pour le peuple français " déclare à cette occasion le colonel Sewell, commandant en chef de la base $n^{\circ} 1^{3}$. Tous les baraquements du camp avaient été décorés de guirlandes, de houx, de branches de gui ou de genêts. On fêta également le 14 juillet et le Memorial Day. Les Américains organisèrent aussi des fêtes sportives. Ainsi des courses de motocyclettes, de voitures et même d'ambulances sont-elles organisées du 30 mai au $1^{\mathrm{er}}$ juin 1919 sur la plage de La Baule pour la plus grande joie des 20000 spectateurs civils présents ${ }^{4}$.

Par ailleurs, des contacts individuels sont noués grâce à des invitations de militaires américains dans des familles nazairiennes ou nantaises pour

2. Ibidem.

3. Le Courrier de Saint-Nazaire, 29 décembre 1917.

4. Le Réveil Artistique, 31 mai 1919. 
un déjeuner ou un dîner. "Certains d'entre nous ont la chance d'être reçus en tels termes d'intimité avec quelques-unes des familles du voisinage que nous pourrions apparaître comme des fils ou des frères, non comme des étrangers fraîchement débarqués " écrit Robert Williams dans le O O la la Times, journal de la garnison, le 25 décembre 1917. Le cercle franco-américain de Nantes - qui a à sa tête le président du Conseil général de la LoireInférieure, Jamin - organise chaque vendredi une soirée dansante!

\section{L'engouement de la population française pour les Sammies}

Il ne fait pas de doute que la population de la Basse-Loire manifeste à de nombreuses reprises sa sympathie pour les Sammies. Les rapports des commissions militaires de contrôle postal ainsi que le « bulletin confidentiel résumant la situation morale à l'intérieur " rédigé par l'État Major de l'Armée, nous renseignent sur l'effet produit sur l'opinion publique de la région par le débarquement des troupes américaines : confiance et espoir sur l'heureuse issue de la guerre se répandent après le pessimisme du printemps et du début de l'été 1917. Les Américains rassurent d'abord par leur présence. Les rapports des commissaires centraux de police et des commissaires spéciaux de Nantes et de Saint-Nazaire sur l'état d'esprit des populations sont très significatifs : "L'arrivée ininterrompue des contingents américains ne fait qu'augmenter la bonne impression des populations et la foi dans l'avenir ${ }^{5}$. " Des sentiments identiques sont d'ailleurs exprimés dans l'ensemble de la France. On compte beaucoup sur leur aide pour mener à bonne fin les opérations militaires. L'opinion publique se rend compte alors que l'aide nécessaire n'est pas aussi platonique ni aussi tardive que ne l'avaient craint pendant un certain temps beaucoup de Français.

Les Américains impressionnent par leur efficacité. La réalisation des camps, voies ferrées, gares de triage en un temps record sur des terrains où il y avait peu de temps auparavant que quelques paisibles troupeaux de chevaux ou de bœufs plonge les Nazairiens dans la stupeur : "Il faut bien le dire, ce que j'ai vu [à Montoir] dépasse tout ce que mon imagination pouvait concevoir. Je suis revenu ravi, enthousiasmé, émerveillé de l'effort énorme, considérable, prodigieux accompli par nos alliés dans notre contrée nazairienne ", écrit Dubois Savary dans Le Courrier de Saint-Nazaire du 21 septembre 1918. Le sous-préfet de Saint-Nazaire, Roland Gaignerot, note en février 1918 que « la présence ici des Américains, le matériel qu'ils débarquent, les travaux qu'ils entreprennent sont des éléments de réconfort pour les Nazairiens qui constatent au premier rang l'effort considérable de nos alliés".

Des réactions identiques sont notées dans d'autres régions de France. Ainsi le rapport mensuel de la commission militaire de contrôle postal de Marseille indique que les méthodes de travail des Américains, rapides, pré-

5. Arch. dép. de Loire-Atlantique, 1 M 529, rapport mensuel du commissaire central de police de Nantes, 18 mars 1918. 
cises, le dédain qu'ils ont pour la paperasserie et les formalités administratives surprennent agréablement. Le matériel et l'esprit d'organisation des Américains éblouissent les Français ${ }^{6}$. L'apparence physique des Sammies impressionne. Ce sont " des hommes forts et jeunes", de "solides gaillards ", "tous jeunes et magnifiques "! Les habitants de la BasseLoire découvrent chez les Américains de grandes qualités. Tout d'abord la majorité de ces derniers se conduit de façon correcte et accepte de bon cœur la discipline. Le bulletin confidentiel de l'État-Major de l'Armée, consacré à la XI Région, signale par exemple en juillet 1918 que " la tenue des Américains " attire " la sympathie et l'admiration de la population ${ }^{7}$ ". On vante aussi leur sens de l'épargne. Les dockers noirs américains se sont tous fait ouvrir un compte en banque. Les journaux se font d'ailleurs l'écho de la stupéfaction que provoque les premières fois l'arrivée de dockers en bourgeron de toile bleue au milieu des hommes d'affaires et des négociants du Crédit Orléanais à Nantes ${ }^{8}$.

On apprécie aussi la "simplicité directe ", la " modestie dans le comportement " des soldats américains ${ }^{9}$. Un journaliste du Phare de la Loire décrit les Américains du camp d'hydravions du Croisic comme particulièrement " affables et serviables ", très " estimés " par la population, par opposition aux militaires français du camp voisin, dépeints comme hautains, arrogants et pleins de morgue ${ }^{10}$. Une Nazairienne, Madame Manet, qui de plus a voyagé aux États-Unis, s'émerveille de la "joie d'enfants " des Sammies, espiègles et insouciants. Ils sont de bonne humeur et ont le sens de l'humour ${ }^{11}$. Ils manifestent un " grand amour des enfants " qu'ils " comblent de friandises ${ }^{12}$ ".

La plus grande partie de la population est saisie d'un extraordinaire engouement pour les Américains. On espère que les qualités des Américains déteindront sur les Français, en particulier pour modifier les procédés routiniers des administrations françaises et pour acquérir davantage de sens pratique et de goût pour l'action. Certains journalistes préconisent même une "cure d'américanisme ". D'une manière plus superficielle, l'Amérique est à la mode. Des hôtels changent de nom. Ainsi, Saint-Nazaire a son American Hotel et Pornichet son Select Hotel. Cinémas et théâtres affichent fréquemment des œuvres évoquant l'Amérique, soit par le titre, soit par leur action, soit par leurs personnages. La mode ellemême subit l'influence américaine : des femmes adoptent des chapeaux à

6. KASPI, André, Le Temps des Américains, 1917-1918, Paris, Publications de la Sorbonne, 1976, p. 129.

7. Arch. du ministère de la Guerre (SHAT), E.M.A. 174/1, cabinet du Ministre (cabinet militaire).

8. Le Télégramme des Provinces de l’Ouest, 23 juillet 1917.

9. Le Courrier de Saint-Nazaire, Le Phare, Le Populaire.

10. Arch. dép. de Loire-Atlantique, 1 M 659-660. L'article fut arrêté par la censure étant donné le jugement sévère porté sur une unité française.

11. The Gangplank News, 14 avril 1919.

12. Nantes Mondain, 19 janvier 1918. 
larges bords inspirés de ceux des Sammies, les jeunes gens se font raser les moustaches et tailler les cheveux "à l'américaine ${ }^{13}$ ". L'étude de dessins d'enfants réalisés dans des écoles nantaises pendant la guerre confirme ces impressions. La présence des Sammies est l'objet de curiosité pour les écoliers qui découvrent le chewing gum - que les élèves appellent le " chien gomme " - la "musique nègre " et les puissants camions qui font trembler le sol ${ }^{14}$.

Il ne faudrait pourtant pas croire que l'harmonie soit totale entre soldats américains et civils français. La présence des Sammies entraîne certains désagréments : routes défoncées par les allées et venues des camions, occupations de terrains ou de locaux, brutalités commises par certains soldats. Les Français reprochent aux Américains de dépenser trop facilement et donc de faire augmenter le coût de la vie. De plus, les Français accusent les nouveaux arrivants de s'intéresser un peu trop aux femmes. Mais c'est surtout à partir de novembre 1918 que certaines gênes causées par la présence américaine sont considérées comme insupportables et que de nouveaux motifs de plaintes apparaissent.

\section{La dégradation des rapports entre civils français et militaires américains}

Il faut tout d'abord reconnaître que la discipline s'est relâchée dans l'armée américaine avec la fin des combats. Ce ne sont plus les mêmes troupes qui séjournent dans la Basse-Loire. Désormais, ce sont presque uniquement des troupes de passage, pressées de se rembarquer alors que les soldats " sédentaires " - tels ceux du 7th Engineers - avaient fini par se sentir totalement intégrés à la région. Le commissaire de police de SaintNazaire s'adresse le 14 décembre 1918 au préfet de la Loire-Inférieure en ces termes:

" Depuis une date qui se rapprocherait de la signature de l'armistice, les petits conflits, d'ailleurs fort rares, qui avaient pu s'élever dans la région de Saint-Nazaire, entre la population et les militaires alliés se sont multipliés et aggravés ${ }^{15}$."

D'une façon générale, les journaux locaux rapportent volontiers les incidents entre la population et l'armée alliée. Certes, la plus grande souplesse de la censure après l'armistice permettait tout naturellement de relater les incidents qu'il fallait auparavant passer sous silence. On a toutefois l'impression que les journaux insistent sur ce type d'événements pour plaire à leurs lecteurs. Certains incidents prennent un aspect plus sérieux. Ainsi, le 6 avril 1919, se produit à Saint-Nazaire une véritable manifestation anti-américaine. Les journaux, notamment Le Phare de la Loire, font état de bagar-

13. L'Express de l'Ouest, 11 juillet 1917.

14. GuYvarc'H, Didier, Moi Marie Rocher, écolière de guerre. Dessins d'enfants, 1914-1919, Rennes, Apogée, 1993, p. 67-72.

15. Arch. dép. de Loire-Atlantique, 2 R 369, base américaine, affaires diverses. 
res, d'actes de violence où sont impliqués des soldats américains. On parle d'une " ambiance de western "! Des policiers américains ont dû tirer des coups de feu en l'air place Carnot et rue de Nantes et la police française a dû intervenir également. Au total, six blessés - des civils français - ont été transportés à l'hôpital ${ }^{16}$. Dans les jours qui suivent, des officiers américains se plaignent d'être frappés ou injuriés lors de leurs allées et venues en ville.

On peut se demander si la patience des Français envers les Américains n'a pas brusquement diminué ou même disparu le 11 novembre 1918. C'est en tout cas l'avis d'un journaliste du Travailleur de l'Ouest - journal de la fédération socialiste de la Loire-Inférieure - qui écrit le 8 février 1919 :

«Tant qu'a duré la guerre, nous avons supporté la présence de nos alliés, surtout les commerçants. La guerre finie, va-t-on leur faire comprendre qu'ils sont indésirables? Je le crains."

Faut-il voir dans cette évolution les résultats d'une " campagne antiaméricaine " organisée « en haut lieu " comme le propose Le Travailleur de l'Ouest à ses lecteurs le 24 mai 1919? Y aurait-il une volonté délibérée des autorités de marquer leur mauvaise humeur devant les divergences de vues qui se sont produites à la Conférence de la Paix à Paris, entre Clemenceau et Wilson? En réalité, il n'en a rien été, bien au contraire. Le gouvernement français comme les autorités locales sont très soucieux de maintenir d'excellents rapports avec les Américains. Ce qui est possible, en revanche, c'est que l'opinion française ait quelquefois traduit, peut-être d'une manière plus ou moins inconsciente, par son comportement envers les Américains, l'hostilité qu'elle manifestait envers les positions américaines à la Conférence de la Paix. Différents journaux parlent en avril 1919 du « malentendu franco-américain ". La presse locale, à l'exception du Travailleur de l'Ouest, qui n'a d'ailleurs qu'une audience limitée, publie des articles hostiles au président Wilson et à ses compatriotes. Certains articles sont même censurés. Une instruction confidentielle du président du Conseil et ministre de la Guerre Clemenceau adressée au général commandant la XI Région le 7 avril 1919 insiste sur la nécessité de maintenir les meilleures relations possibles avec les troupes américaines ${ }^{17}$.

Il n'en reste pas moins que les relations entre civils français et soldats américains ne sont plus les mêmes en 1919 qu'elles l'étaient en 1917 et 1918. Des enquêtes policières menées dans les chantiers de construction navale de Penhoët, aux chantiers de la Loire, le long des docks et dans les cafés, le montrent bien ${ }^{18}$. Il en est d'ailleurs de même dans d'autres régions de France. Ainsi, des accrochages se produisent entre Américains et Français à Brest en juin, juillet et août $1919^{19}$. Les facilités pécuniaires des

16. Arch. dép. de Loire-Atlantique, 1 M 529, rapport du commissaire central de police de Saint-Nazaire et du sous-préfet de Saint-Nazaire au préfet de Loire-Inférieure, 17 avril 1919.

17. Arch. dép. de Loire-Atlantique, 1 M 659-660.

18. Arch. dép. de Loire-Atlantique, 2 R 372.

19. SHAT, E.M.A., 174/1, cabinet du ministre (cabinet militaire), " bulletin confidentiel résumant la situation morale à l'intérieur ". 
Américains provoquent des jalousies. Les journaux locaux dénoncent, parfois de façon véhémente, la flambée des prix ou en rendent souvent responsables les soldats américains. Le commissaire spécial de Saint-Nazaire signale dans ses rapports au sous-préfet que la présence des troupes américaines dans l'arrondissement a eu pour conséquence immédiate " l'augmentation du prix de la vie ", essentiellement le prix des denrées et celui des loyers ${ }^{20}$. Le président du Conseil, ministre de la Guerre, a sur le sujet un échange de correspondance avec le général Pershing, commandant en chef des Forces expéditionnaires américaines, à la fin de 1917 et au début de $1918^{21}$. Une enquête effectuée à la fin de 1917 par l'intendant militaire Péria permet de prouver qu'effectivement certaines denrées alimentaires ont subi une hausse considérable dans les régions où stationnaient des troupes américaines. La prodigalité naturelle des soldats américains, leur solde relativement élevée, leur mauvaise connaissance de la langue française et l'âpreté au gain de certains commerçants peuvent expliquer certains dérapages. Cependant, la presque totalité des augmentations de prix a été provoquée par les mêmes causes générales que dans toute la France. Il ne faut pas oublier que les Américains faisaient venir d'Amérique tous les produits alimentaires dont ils avaient besoin. Si les soldats américains pratiquaient des achats, c'était pour compléter la nourriture qu'ils recevaient dans les camps ou satisfaire leur gourmandise. Cela ne portait donc que sur des produits particuliers tels que beurre, œufs, fromage, légumes verts et fruits. Les Américains se précipitent aussi sur les alcools et consomment beaucoup de pain. La présence d'effectifs américains assez considérables et l'installation de nombreux services absorbèrent toutes les disponibilités en logements de Nantes et Saint-Nazaire. Le sous-préfet de Saint-Nazaire estime le 15 juin 1919 que la crise des logements durera " tant que les Américains conserveront leur base de Saint-Nazaire ${ }^{22}$ ".

L'attitude des Américains à l'égard des femmes a également contribué à créer des tensions, surtout à partir de l'armistice. La présence des soldats alliés avait donné naissance à des trafics de toutes sortes, et notamment à un développement de la prostitution. L'arrivée des troupes américaines avait attiré, dans toutes les localités occupées, une quantité de filles alléchées par l'espoir d'un gain facile. Ainsi en dehors des maisons publiques proprement dites, il y eut à Saint-Nazaire plus de 300 cafés qui jouèrent le rôle de maisons publiques clandestines. Le racolage s'exerçait sur le trottoir ${ }^{23}$. Il en est de même à Nantes. "Les soldats américains racolent tout ce qu'ils trouvent " note le commissaire central de cette ville ${ }^{24}$. Un rapport du général Coutanceau signale en janvier 1919 que la dépravation va en augmentant dans les zones de Nantes et de Saint-Nazaire ${ }^{25}$. Le

20. Arch. dép. de Loire-Atlantique, 1 M 529, rapport du 18 août 1918.

21. SHAT, E.M.A., 4855, bureau spécial franco-américain.

22. Arch. dép. de Loire-Atlantique, 2 R 372.

23. SHAT, mission militaire française près l'armée américaine, carton $n^{\circ} 10$.

24. SHAT 7 N 2256, rapport du 25 octobre 1918.

25. Arch. dép. de Loire-Atlantique, 2 R 372, rapport du 30 janvier 1919. 
Courrier de Saint-Nazaire du 24 mai 1919, dans un article intitulé " un coup de balai SVP " constate que "le dévergondage des mœurs est devenu si impudent à Saint-Nazaire qu'il s'affiche au grand jour ". Des rapports de police français font état d'une recrudescence des maladies vénériennes et préconisent que les autorités militaires américaines reviennent sur l'interdiction faite aux soldats de fréquenter les maisons de tolérance. Mais ces mêmes autorités américaines estiment que celles-ci n'offrent pas les conditions voulues d'hygiène et de propreté.

Par ailleurs, les soldats français rentrant du front après l'armistice, voient d'un mauvais œil l'engouement des jeunes filles françaises pour les soldats américains. Le Courrier de Saint-Nazaire publie plusieurs lettres de " poilus ", assez acides à l'égard des Sammies et de leurs petites amies. L'un d'entre eux reproche aux jeunes filles un " laisser-aller de mauvais goût ". La chaude camaraderie des combattants unis contre l'ennemi commun semble brusquement évanouie devant les conquêtes féminines réalisées par les Américains ${ }^{26}$.

Un nombre non négligeable de mariages furent conclus entre de jeunes Françaises et des Américains, surtout en 1919 : 175 à Saint-Nazaire, 74 à Nantes. Les mariages franco-américains n'ont pas été au total tellement nombreux (environ 320). Ils n'en constituent pas moins, dans certains cas, un pourcentage important du nombre total des mariages, surtout en 1919. Ainsi 22 \% des mariages à Saint-Nazaire et à Montoir, 19 \% à Savenay sont franco-américains. Certains mois de l'année 1918 ou de l'année 1919, le pourcentage des mariages franco-américains à Saint-Nazaire est vraiment impressionnant : $30 \%$ en janvier 1918, $34 \%$ en mai $1919,37 \%$ en juin ${ }^{27}$. Les mariages franco-américains furent-ils réussis? Il est assez difficile de répondre à cette question car un certain nombre de divorces prononcés en Amérique n'ont pas été reportés sur les registres français. Une malicieuse remarque du Réveil Artistique du 28 décembre 1918 indique quelle a pu être la source de certaines déceptions :

"On dit que nos charmantes nazairiennes qui ont épousé des militaires américains sont sûres d'accompagner leur mari aux États-Unis et de trouver leur home luxueusement installé dans la Cinquième Avenue de New York! ”

Un certain nombre de fiancées ne partirent jamais avec l'Américain qui leur avait promis le mariage. D'autres, une fois mariées et parties aux ÉtatsUnis, revinrent de là-bas désenchantées.

S'il est incontestable que l'attitude de la population française - avec l'exception des épisodes des mariages - devient plus hostile vis-à-vis des soldats américains à partir de la fin de 1918, le même phénomène s'observe en sens inverse. Les signes de désaffection à l'égard de la France n'affectent d'ailleurs pas seulement les unités stationnées en Loire-Inférieure mais l'ensemble de l'armée américaine en France puisqu'ils sont signalés à la fois par

26. Le Courrier de Saint-Nazaire, 22 février - $1^{\mathrm{er}}$ mars, 2 mars et 15 mars 1919.

27. Registres de l'État-civil des communes de Saint-Nazaire, Nantes, Montoir et Savenay. 
l'attaché militaire français à Washington et par le maréchal commandant les armées françaises de l'Est ${ }^{28}$. Beaucoup de soldats américains retournent chez eux dans un état d'irritation inquiétant pour la bonne renommée de la France aux États-Unis. Ce sont surtout les exigences des petits commerçants français qui ont exaspéré les soldats américains ainsi qu'en témoigne cet article publié dans le New York Tribune le 17 février 1919 :

"Nous sommes venus ici pour nous battre pour la France, nous nous sommes battus et avons fait de notre mieux pour elle, et en échange nous avons été exploités par un peuple rapace qui n'a vu en nous qu'une source de dollars. "

Un article de Wimfred Black dans le New York American accuse les Français d'avoir exploité les Américains ${ }^{29}$. Les autorités françaises s'inquiètent de l'image que les Sammies garderont des Français. Le général commandant la XI ${ }^{\mathrm{e}}$ Région est invité à faire des visites officielles aux autorités américaines pour leur exprimer « les sentiments de gratitude et de sympathie de la France ". Des réceptions et des manifestations de sympathie avec musique militaire, fleurs, envoi de délégations et de détachements de troupe accompagnent le départ des bateaux ${ }^{30}$. Les municipalités de Nantes et de Saint-Nazaire font également tout leur possible pour maintenir et renforcer les relations amicales entre Français et Américains. Par exemple, le maire de Nantes, Paul Bellamy, écrit au président du Comité franco-américain le 19 février 1919 pour lui dire que " la ville de Nantes désire pouvoir manifester à nouveau les sentiments de cordiale sympathie qui l'unissent à ses alliés américains en s'associant à la fête organisée par le Comité franco-américain de Nantes, à l'occasion de l'anniversaire de George Washington ${ }^{31}$ ". En avril 1919, le maire de Saint-Nazaire affirme que les autorités américaines, les pouvoirs publics et la municipalité ne manqueront pas de s'employer de toutes leurs forces pour assurer la sympathie des relations entre les soldats américains et la population française, " sympathie que nous désirons voir exister et persister entre nos alliés et nous ${ }^{32}$ ". Il ne fait aucun doute que les autorités ont tout fait pour maintenir la cordialité des rapports avec les Américains.

Il ne faut donc pas pousser au noir la peinture des relations entre soldats américains et civils français en 1919. En réalité, si le contraste est net avec les deux années précédentes, il est loin d'être brutal. L'évolution n'a été ni générale, ni soudaine. Aussi, des manifestations de sympathie en faveur des Sammies se produisent en Basse-Loire après le 11 novembre 1918 et jusqu'en octobre 1919, date du départ des derniers soldats américains, mais l'atmosphère chaleureuse et euphorique des premiers temps avait disparu.

28. SHAT, E.M.A., 4856, bureau spécial franco-américain.

29. Cité par l'Exportateur français, 3 avril 1919.

30. Arch. dép. de Loire-Atlantique, 1 M 659-660, instruction du 7 avril 1919.

31. Arch. dép. de Loire-Atlantique, 2 R 409.

32. Arch. dép. de Loire-Atlantique, 1 M 529, lettre au sous-préfet de Saint-Nazaire. 


\section{Une influence finalement limitée sur l'évolution de la Basse-Loire}

Au lendemain de la guerre, les responsables des ports de la Basse-Loire espéraient que l'importance du trafic du temps de guerre contribuerait à faire naître des courants commerciaux durables avec les États-Unis. Dans une interview accordée à un journaliste en janvier 1918, le nouveau consul général des États-Unis à Saint-Nazaire, Ravndal, avait affirmé que son plus grand désir était de contribuer de toutes ses forces " à l'extension des relations économiques entre la région de la Loire et les États-Unis d'Amérique $^{33}$ ". Le ministre du Commerce lui-même, Clémentel, avait déclaré en décembre 1918 que l'estuaire de la Loire où de grands travaux avaient été exécutés pendant la guerre, se prêtait particulièrement bien aux échanges commerciaux avec les États-Unis ${ }^{34}$. Certes, la région a bénéficié de certains travaux réalisés par les Américains, notamment en matière ferroviaire. Mais les grands espoirs de créer des relations commerciales durables avec les États-Unis ne verront pas le jour. Le grand projet de liaison Suisse - Océan qui consistait pour les ports de Nantes et de SaintNazaire de détourner à leur profit une partie du trafic des ports allemands de Hambourg et de Brême et de devenir ainsi la voie d'entrée des marchandises à destination de la Suisse et de l'Europe centrale, ne fut jamais réalisé. Il en fut de même pour les échanges avec les États-Unis.

Ce ne sont pas des raisons nautiques qui ont amené les Américains à renoncer à établir les relations commerciales avec la Basse-Loire. Nantes et Saint-Nazaire sont des ports bien aménagés et bien outillés, mais ils subissent la concurrence victorieuse d'autres ports français ou étrangers : l'ancienneté et la puissance des courants commerciaux attiraient au Havre ou dans les ports de la mer du Nord (Anvers, Rotterdam), le trafic des ÉtatsUnis avec l'Europe. Les facteurs économiques essentiels que sont la situation géographique - c'est-à-dire la distance par rapport aux gros centres de clientèle - et les diverses qualités techniques qui se traduisent par l'établissement des frais de port, expliquent que d'autres ports aient été choisis par les Américains. La fréquence des départs ou des arrivées des navires, les garanties boursières, financières, juridiques des contrats ont déterminé les choix américains ${ }^{35}$. Il faut ajouter que les industries de la région ne réclamaient pas spécialement de produits américains et que les produits locaux n'étaient pas susceptibles d'alimenter un grand trafic d'exportation.

Sur le plan humain, Nantais et Nazairiens avaient pu apprécier l'efficacité des méthodes américaines. La présence des Sammies n'était pas passée inaperçue. Leur nombre, leur activité, leur comportement contribuèrent à changer la physionomie des localités où ils étaient installés. " Pour un peu, ils eussent défriché notre vieille cité comme une terre neuve ou

33. La Loire navigable, janvier-mars 1918.

34. Le Télégramme des Provinces de l'Ouest, 5 janvier 1919.

35. VIGARIE, André, Les grands ports de commerce de la Seine au Rhin, Paris, FABRI, 1964. 
une forêt du nouveau monde " remarquait Rondeau, secrétaire général de la ville de Nantes. En fait, l'« américanisation » n'apparaît pas très profonde. Malgré une imitation parfois même poussée à l'excès, les Français ne semblent pas avoir transformé réellement leur mode de vie ou leur comportement. Il faut bien dire que si les soldats américains aiment à flâner à leurs moments de loisir dans les rues de Nantes ou de Saint-Nazaire et apprécient les rencontres individuelles, ils se mêlent peu en tant que collectivité à la vie des deux cités. L'essentiel de leurs activités se déroule dans le cadre militaire américain. Ils éditent des journaux, pratiquent leurs sports nationaux, conservent leurs traditions religieuses comme s'ils avaient été en Amérique. Nantais et Nazairiens, dont la vie est certes quelque peu bouleversée par la présence des Américains, se contentent souvent d'être de simples spectateurs.

L'existence de ces masses importantes de soldats à proximité de populations civiles ne fut pas, nous l'avons vu, sans poser quelques problèmes. Après une période d'enthousiasme, les qualités qu'on avait admiré chez les Américains s'étaient retournées contre eux : leur richesse et leur efficacité n'étaient plus admirées, mais enviées, jalousées. On craignait la concurrence et la réussite de ces trop puissants alliés. La menace allemande une fois écartée, la gêne inévitable que provoque la présence de forces militaires étrangères, même amies, même alliées, entraîne des réactions négatives de la part de la population civile française.

\section{Soldats américains défilant dans une rue de Saint-Nazaire (@ BNF)}

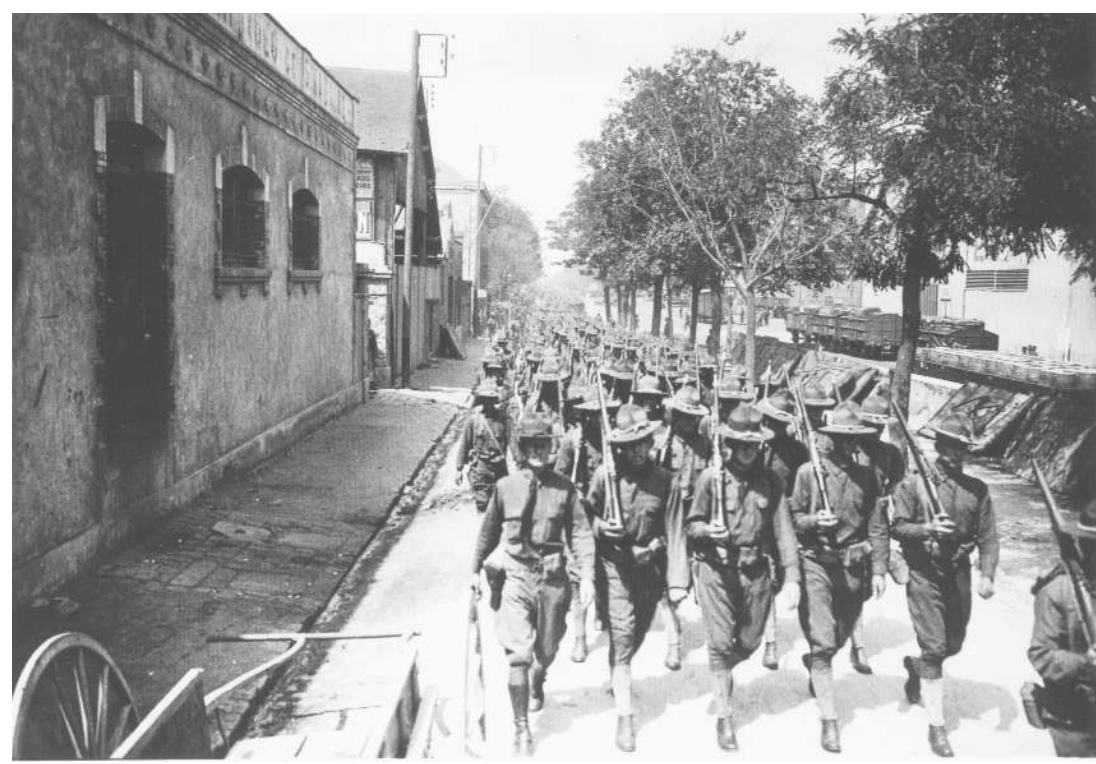


Camions et soldats américains sur le port de Saint-Nazaire (@ BNF)

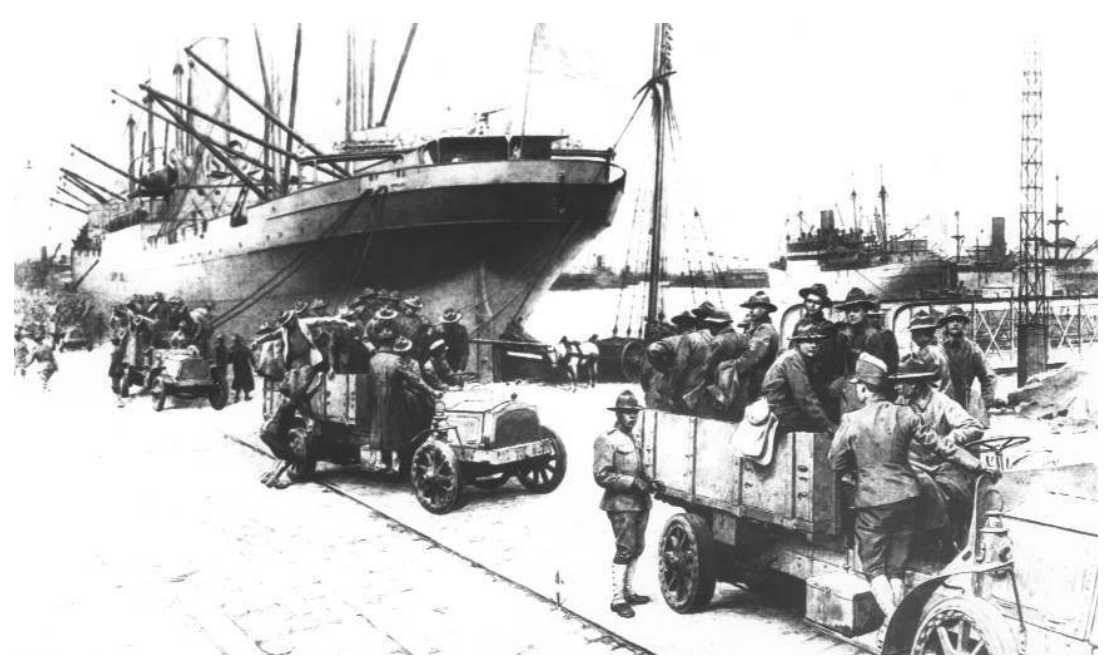

Troupes américaines sur les quais de Saint-Nazaire après leur débarquement d'un bateau (@ BNF)

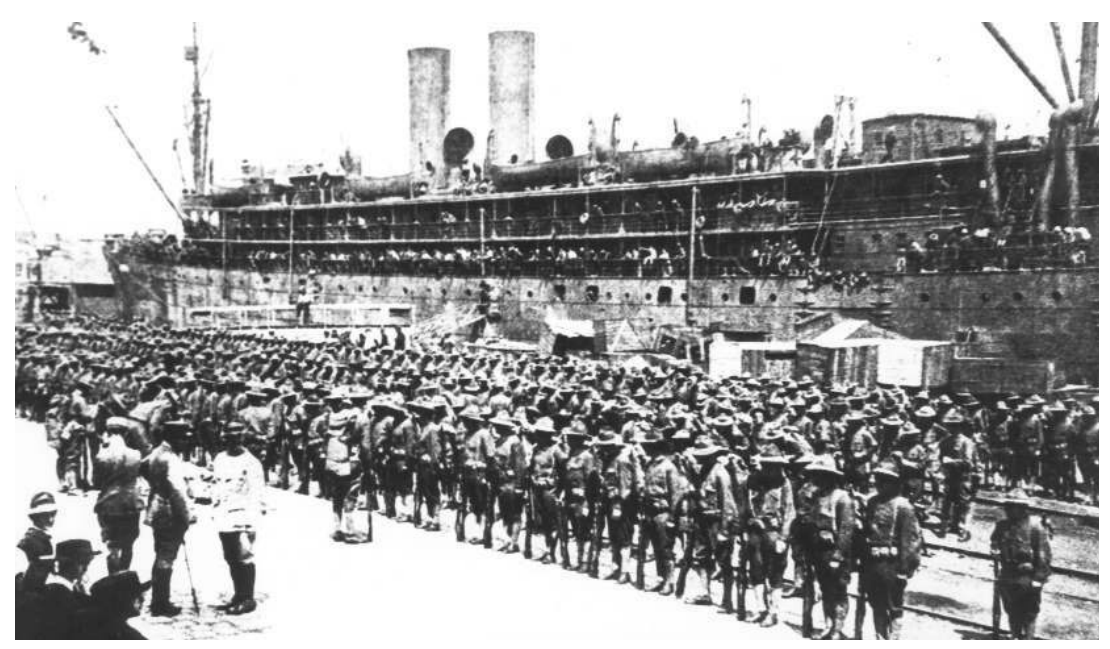




\section{RESUME}

La région de la Basse-Loire, Base $n^{\circ} 1$ du Corps expéditionnaire américain pendant la Première Guerre mondiale voit débarquer 198000 hommes en 1917-1918 tandis que 478000 repartent par Saint-Nazaire de janvier à octobre 1919. Selon les moments, 15000 à 60000 Sammies séjournent dans la région.

La population locale exprime sa sympathie et sa reconnaissance envers ces " sauveurs " arrivés au bon moment pour épauler les " poilus ". On admire leur efficacité, leur sens de l'organisation, leur bonne humeur, leur simplicité.

La menace allemande une fois écartée, après le 11 novembre 1918, la gêne inévitable que provoquait la présence de cette force militaire étrangère, même amie, même alliée, entraîne des réactions négatives de la part de la population civile française.

\section{ABSTRACT}

The Basse-Loire region, the Base 1 of the American Expeditionary Forces during the First World War, sees the landing of 198,000 men in 1917-1918, while 478,000 leave via Saint-Nazaire from January to October of 1919. At one time or another, 15,000 to 60,000 Sammies stay in the region.

The local population expresses their sympathy and gratitude towards these "saviors" arriving at just the right moment to support the "poilus". One admires their efficiency, their organizational sense, their good mood, and their simplicity.

Once free of the German threat after 11 November 1918, the inevitable discomfort provoked by the presence of this foreign military force - even an allied and friendly one-leads to negative reactions on the part of the French civil population. 